\title{
Dedekind and Hardy sums
}

R. Sitaramachandrarao* (Toledo, Ohio)

In memoriam G. H. Hardy

1. Introduction. The Dedekind sum $s(h, k)$, arising in the theory of the Dedekind eta function, is defined by

$$
s(h, k)=\sum_{\mu(\bmod k)}\left(\left(\frac{\mu}{k}\right)\right)\left(\left(\frac{h \mu}{k}\right)\right)
$$

where $h$ is an integer and $k$ is a positive integer and

$$
((x))= \begin{cases}x-[x]-\frac{1}{2} & \text { if } x \text { is not an integer, } \\ 0 & \text { otherwise }\end{cases}
$$

$[x]$ being the largest integer $\leqslant x$.

The most important property of Dedekind sums is the following reciprocity theorem. If $h$ and $k$ are coprime positive integers, then

$$
s(h, k)+s(k, h)=-\frac{1}{4}+\frac{1}{12}\left(\frac{h}{k}+\frac{k}{h}+\frac{1}{h k}\right) .
$$

Dedekind [15] was the first to give a proof of (1.1) based on his Dexist several proofs of transformation formula for $\log \eta(\tau)$. At present, there exist several proofs monograph of $H$. Rademacher and E. Grosswald [26], Berndt [3], [6], [7] and U. Dieter [16].

Apparently, G. H. Hardy [19], in 1905, was the first to give a proof of he reciprocity theorem for Dedekind sums which does not depend on the theory of the $\eta$-function. In fact, Hardy, using contour integration, proved some reciprocity theorems in detail and, at the end of the paper, stated eleven more reciprocity theorems for some similar arithmetical sums. One of these is the reciprocity theorem for Dedekind sums (cf. [19], eqn. (iii) on p.

\footnotetext{
* On leave from Andhra University, Waltair, India.
} 
122). Also Hardy clearly indicated the necessary modifications in his foregoing proof to obtain these reciprocity theorems.

In recent years, five of Hardy's reciprocity theorems (capsulized into four) were found in an interesting way by B. C. Berndt [8], and L. A. Goldberg [18]. Just as Dedekind deduced (1.1) from his transformation formula for $\log \eta(\tau)$, Berndt [8] and Goldberg [18] encountered these sums (in a slightly different notation) and deduced these reciprocity theorems from Berndt's transformation formulae [8] for the logarithms of the classical theta-functions $\theta_{2}(0, q), \theta_{3}(0, q)$ and $\theta_{4}(0, q)$ (cf. [29], Chapter 21). It may be of interest to note that, in his introduction, Hardy (cf. [19], p. 94, lines 14-18) observes "...though they are in reality limiting cases of the formulae of the transformation theory or of formulae deducible from them..."

L. A. Goldberg [18] showed that these sums also arise in the theory of $r_{s}(n)$, the number of representations of $n$ as a sum of $s$ integral squares and in the study of the Fourier coefficients of the reciprocals of $\theta_{n}(0, q), n=2,3,4$ while B. C. Berndt and L. A. Goldberg [12] (in the spirit of B. C. Berndt [5]) evaluated certain non-absolutely convergent double series, in closed form, in terms of these sums.

Since Hardy was the first to encounter these sums and formulated these reciprocity theorems with clear indications of proofs, we will refer to these, in the sequel, as. Hardy sums and Hardy's reciprocity theorems.

At the end of his paper, Hardy states "...I hope on some other occasion to return to these formulae from an arithmetical point of view..." but it appears that Hardy never returned to the subject. In recent years, proofs of Hardy's reciprocity theorems which do not depend on Berndt's transformation formulae were given by T. M. Apostol and T. H. Vu [1], B. C. Berndt and L. A. Goldberg [12], B. C. Berndt and U. Dieter [10], U. Dieter [16] and B. Davis and the author [14].

The object of this paper is (a) to give an elementary proof of Dedekind's [15] infinite series representation for $s(h, k)$, namely that, for $(h, k)=1$

$$
s(h, k)=\frac{1}{2 \pi} \sum_{\substack{r=1 \\ r \neq 0(\bmod k)}}^{\infty} \frac{\cot (\pi h r / k)}{r}
$$

(b) to deduce Dedekind's reciprocity theorem (1.1) from Dedekind's representation (1.2) and give elementary proofs, based on (1.2), of $P$. Subrahmanyam's identity [28] and M. I. Knopp's theorem [20] for Dedekind sums (c) to express, using elementary arguments, each one of Hardy sums explicitly in terms of Dedekind sums (d) to deduce, elementarily, Hardy's reciprocity theorems from our explicit formulae and (1.1) and (e) to deduce B. C. Berndt and L. A. Goldberg's [12] and L. A. Goldberg's [18] analogues, for Hardy sums, of certain results due to Dedekind, Rademacher, Subrahmanyam and Knopp. We believe that our explicit formulae will be useful in the study of congruence properties of Hardy sums and construction of tables for Hardy sums.

The reader of Hardy's paper should note that Hardy uses

$$
\begin{aligned}
& \varepsilon_{x} \text { for } \begin{cases}(-1)^{[x]} & \text { if } x \text { is not an integer and } \\
0 & \text { otherwise and }\end{cases} \\
& {[x] \text { for }\left(\left(x+\frac{1}{2}\right)\right)}
\end{aligned}
$$

where $[x]$ and $\left(\left(x+\frac{1}{2}\right)\right)$ appearing on the right of above are in the notation of this paper.

The author is highly thankful to Prof, D. H. Lehmer, Prof. E. Grosswald and Prof. M. I. Knopp for their encouragement and criticism and to Prof. T. M. Apostol, Prof. Bruce C. Berndt and Dr. L. A. Goldberg for making available various of their works. Finally, the author wishes to thank the referee for a critical reading and useful advices.

2. Dedekind's representation. In this section, using finite Fourier series, we prove Dedekind's infinite series representation (1.2) for $s(h, k)$. For another proof, we refer to B. C. Berndt (cf. [6], Lemma 2.1) which uses Fourier series. Using finite Fourier series, H. Rademacher (cf. [24] and [25], p. 18) proved that for $(h, k)=1$

$$
s(h, k)=\frac{1}{4 k} \sum_{r=1}^{k-1} \cot \left(\frac{\pi r}{k}\right) \cot \left(\frac{\pi h r}{k}\right)
$$

and thus (1.2) is equivalent to

$$
\sum_{\substack{r=1 \\ r \neq 0(\bmod k)}}^{\infty} \frac{\cot (\pi h r / k)}{r}=\frac{\pi}{2 k} \sum_{r=1}^{k-1} \cot \left(\frac{\pi r}{k}\right) \cot \left(\frac{\pi h r}{k}\right) .
$$

This follows readily from the following more general

LEMMA 2.1. Let $f(r),-\infty<r<\infty$, be a complex valued periodic sequence of $a$ period $k$ and be odd. Then

$$
\sum_{r=1}^{\infty} \frac{f(r)}{r}=\frac{\pi}{2 k} \sum_{r=1}^{k-1} f(r) \cot \left(\frac{\pi r}{k}\right)
$$

Proof. Following D. H. Lehmer [21], we define the Euler constant $\gamma(r, k)$, associated with the arithmetical progression $r, r+k, r+2 k, \ldots$, $1 \leqslant r \leqslant k$, by

$$
\gamma(r, k)=\lim _{x \rightarrow \infty}\left(\sum_{\substack{0<n \leqslant x \\ n \equiv r(\bmod k)}} \frac{1}{n}-\frac{1}{k} \log x\right) .
$$


Using finite Fourier series, D. H. Lehmer [21] proved that

$$
\gamma(k-r, k)=\gamma(r, k)-\frac{\pi}{k} \cot \frac{\pi r}{k}, \quad 1 \leqslant r \leqslant k-1
$$

and also that if $g(r),-\infty<r<\infty$, is any complex valued sequence of period $k$, then

$$
S(g) \equiv \sum_{r=1}^{\infty} \frac{g(r)}{r}=\sum_{r=i}^{k} g(r) \gamma(r, k)
$$

provided $\sum_{r=1}^{k} g(r)=0$; which is a necessary and sufficient condition for convergence of $S(g)$.

To prove Lemma 2.1, we note that $f(k)=0$ and $\sum_{r=1}^{k} f(r)=0$ since $f(r)$ is odd and has a period $k$. Hence by (2.3) and (2.2)

$$
\begin{aligned}
S(f): & =\sum_{r=1}^{\infty} \frac{f(r)}{r}=\sum_{r=1}^{k} f(r) \gamma(r, k) \\
& =\sum_{r=1}^{k-1} f(r) \gamma(r, k)=\sum_{r=1}^{k-1} f(k-r) \gamma(k-r, k) \\
& =-\sum_{r=1}^{k-1} f(r)\left(\gamma(r, k)-\frac{\pi}{k} \cot \left(\frac{\pi r}{k}\right)\right) \\
& =-S(f)+\frac{\pi}{k} \sum_{r=1}^{k-1} f(r) \cot \left(\frac{\pi r}{k}\right)
\end{aligned}
$$

and the lemma follows.

Remark 2.1. For a different formulation of Lemma 2.1, we refer to B. C. Berndt [9]. His proof is based on an interesting technique in contour integration. Probably Lemma 2.1 is an old result.

3. Dedekind's reciprocity theorem. In this section, we give a proof of the reciprocity theorem for Dedekind sums based on Dedekind's representation (1.2) for $s(h, k)$. For another proof of $(1.1)$, based on (1.2), we refer to B. C. Berndt [6] who however used contour integration in the spirit of G. H. Hardy [19].

For positive integers $h, k$ let

$$
B(h, k)=\sum_{\substack{n=1 \\ m \neq \neq n k}}^{\infty} \sum_{m=1}^{\infty} \frac{1}{m^{2} h^{2}-n^{2} k^{2}}
$$

Then it is known (cf. [4], p. 556) that for $(h, k)=1$

$$
B(h, k)=\frac{\pi^{2}}{h k}\left(\frac{1}{4}-\frac{k}{12 h}-\frac{1}{24 h k}+s(h, k)\right) .
$$

However we, using Dedekind's representation (1.2), prove that for $(h, k)=1$

$$
B(h, k)=\frac{\pi^{2}}{h k}\left(\frac{h}{12 k}+\frac{1}{24 h k}-s(k, h)\right) \text {. }
$$

It is clear that the reciprocity theorem for Dedekind sums follows on comparing (3.1) with (3.2).

To prove (3.2), we write for $u>0$

$$
B(u)=\sum_{\substack{m=1 \\ m \neq u}}^{\infty} \frac{1}{m^{2}-u^{2}}
$$

and note that

$$
B(u)= \begin{cases}\frac{1}{2 u^{2}}-\frac{\pi}{2 u} \cot \pi u & \text { if } u \text { is not an integer } \\ \frac{3}{4 u^{2}} & \text { otherwise. }\end{cases}
$$

In fact, the first part is well known. For the second part, we have for an integer $u>0$

$$
\begin{aligned}
B(u) & =-\frac{1}{2 u} \sum_{m=1}^{u-1}\left(\frac{1}{u-m}+\frac{1}{u+m}\right)+\frac{1}{2 u} \sum_{m=u+1}^{\infty}\left(\frac{1}{m-u}-\frac{1}{m+u}\right) \\
& =-\frac{1}{2 u}\left(1+\frac{1}{2}+\ldots+\frac{1}{2 u}-\frac{1}{u}-\frac{1}{2 u}\right)+\frac{1}{2 u}\left(1+\frac{1}{2}+\ldots+\frac{1}{2 u}\right) \\
& =\frac{3}{4 u^{2}}
\end{aligned}
$$

Now, by (1.2),

$$
\begin{aligned}
B(h, k) & =\frac{1}{h^{2}} \sum_{n=1}^{\infty} \sum_{\substack{m=1 \\
m \neq n k / h}}^{\infty} \frac{1}{m^{2}-(n k / h)^{2}} \\
& =\frac{1}{h^{2}} \sum_{\substack{n=1 \\
n \neq 0(\bmod h)}}^{\infty}\left(\frac{h^{2}}{2 n^{2} k^{2}}-\frac{\pi h}{2 n k} \cot \frac{\pi n k}{h}\right)+\frac{1}{h^{2}} \sum_{\substack{n=1 \\
n \equiv 0(\bmod h)}}^{\infty} \frac{3 h^{2}}{4 n^{2} k^{2}} \\
& =\frac{\pi^{2}}{12 k^{2}}\left(1-\frac{1}{h^{2}}\right)-\frac{\pi^{2}}{h k} s(k, h)+\frac{\pi^{2}}{8 h^{2} k^{2}}
\end{aligned}
$$

which is (3.2) and this completes the proof of Dedekind's reciprocity theorem. 
Remark 3.1. B. C. Berndt [4], using (1.1) and (3.1), proved that (3.3)

$$
B(h, k)+B(k, h)=\pi^{2} /(4 h k)
$$

for $(h, k)=1$. It is clear that (3.3) follows from (1.1) and (3.2) and that the double series defining $B(h, k)$ is conditionally convergent. It may be of interest to note that the double series defining $B(1,1)$ was originally considered by G. H. Hardy who also proved that $B(1,1)=\pi^{2} / 8$ and for the details we refer to T. J. I'. A. Bromwich (cf. [13], p. 101, problem 12).

4. Subrahmanyam's identity and Knopp's theorem. As further applications of Dedekind's representation (12), we prove, in this section, an identity due to P. Subrahmanyam [28] and a result due to M. I. Knopp [20] (which was in part conjectured by $\mathrm{H}$. Petersson).

Generalizing a result due to Dedekind (cf. [15], Theorem 2),

P. Subrahmanyam [28] proved the following

THEOREM 4.1. For any positive integer $d$

$$
\sum_{b(\text { mod } d)} s(h+b k, d k)=\sum_{c \mid d} \mu(c) s(h c, k) \sigma\left(\frac{d}{c}\right)
$$

where $\mu$ denotes the Möbius function and $\sigma(n)=\sum_{d ! n} d$.

Theorem 4.1 in case $d=p$, a prime, is due to Dedekind. M. I. Knopp [20] generalized Dedekind's result in a different way and proved

THEOREM 4.2 .

$$
\sum_{\substack{a d=n, b(\bmod d) \\ d>0}} s(a h+b k, d k)=\sigma(n) s(h, k)
$$

L. A. Goldberg [17] noted that one can deduce Knopp's theorem, in an elementary way, from Subrahmanyam's identity. However, the following inversion formula shows that Theorems 4.1 and 4.2 could be deduced from one another by elementary arguments.

LeMMA 4.1 (Inversion Formula). Let $f(m, n)$ and $g(m, n)$ be two complex valued sequences defined for positive integers $m$ and $n$. Then

$$
f(m, n)=\sum_{d \delta=n} g(m d, \delta) \quad \text { for all } m \text { and } n
$$

iff

$$
g(m, n)=\sum_{\mathrm{d} \delta=n} \mu(d) f(m d, \delta) \quad \text { for all } m \text { and } n .
$$

Proof. Assume (4.1). Then

$$
\sum_{d \delta=n} \mu(d) f(m d, \delta)=\sum_{d \delta \neq n} \mu(d) \sum_{a b=\delta} g(m d a, b)
$$

$$
\begin{aligned}
& =\sum_{d a b=n} \mu(d) g(m d a, b)=\sum_{c b=n} g(m c, b) \sum_{d a=\mathfrak{c}} \mu(d) \\
& =g(m, n)
\end{aligned}
$$

which is (4.2). The proof of the second part is similar.

Now on taking

$$
f(h, n)=\sigma(n) s(h, k) \quad \text { and } \quad g(h, n)=\sum_{b(\bmod n)} s(h+b k, d k)
$$

in the above inversion formula, we see that Subrahmanyam's identity implies and is implied by, in an elementary way, Knopp's theorem.

Remark 4.1. Our inversion formula, given in Lemma 4.1, has similar applications in the works of T. M. Apostol and T. H. Vu [2] and L. A. Parson and K. H. Rosen [22].

Now we deduce Theorem 4.1 from (1.2).

LemMA 4.2. Let $(h, k)=1$ and $r \neq 0(\bmod d k)$. Then

$$
\sum_{b(\bmod d)} \cot \left(\frac{\pi r h}{d k}+\frac{\pi r b}{d}\right)=d \cot \left(\frac{\pi r h}{k(r, d)}\right) .
$$

Proof. It is well known (cf. [26], p. 18, Lemma 3) that for non-integral $x$

$$
\sum_{m=1}^{k} \cot \left(\frac{m+x}{k} \pi\right)=k \cot (\pi x) .
$$

Hence on writing $(r, d)=u, r=u r_{1}, d=u d_{1}$ with $\left(d_{1}, r_{1}\right)=1$, we have

$$
\begin{aligned}
\sum_{b(\bmod d)} \cot \left(\frac{\pi r h}{d k}+\frac{\pi r b}{d}\right) & =\sum_{b\left(\bmod u d_{1}\right)} \cot \left(\frac{\pi r_{1} h}{d_{1} k}+\frac{\pi r_{1} b}{d_{1}}\right) \\
& =u \sum_{b\left(\bmod d_{1}\right)} \cot \left(\frac{\pi r_{1} h}{d_{1} k}+\frac{\pi b}{d_{1}}\right)=u d_{1} \cot \left(\frac{\pi r_{1} h}{k}\right)
\end{aligned}
$$

and the lemma follows.

LemMa 4.3. For $(h, k)=1$, we have

$$
\sum_{\substack{r=1,(r, d)=1 \\ r \neq 0(\bmod k)}}^{\infty} \frac{\cot (\pi r h / k)}{r}=2 \pi \sum_{c \mid d} \frac{\mu(c) s(h c, k)}{c}
$$

Proof. By (1.2), we have

$$
\sum_{\substack{r=1,(r, d)=1 \\ r \neq 0(\bmod k)}}^{\infty} \frac{\cot (\pi r h / k)}{r}=\sum_{\substack{r=1 \\ r \neq 0(\bmod k)}}^{\infty} \frac{\cot (\pi r h / k)}{r} \sum_{\substack{c|r \\ c| d}} \mu(c)
$$




$$
\begin{aligned}
& =\sum_{c \mid d} \mu(c) \sum_{\substack{s=1 \\
s c \neq 0(\bmod k)}}^{\infty} \frac{\cot (\pi h s c / k)}{s c} \\
& =\sum_{c \mid d} \frac{\mu(c)}{c} \sum_{\substack{s=1 \\
s \neq 0\left(\bmod \frac{k}{(k, c)}\right)}}^{\infty} \frac{\cot \left(\frac{\pi s h c /(k, c)}{k /(k, c)}\right)}{s} \\
& =2 \pi \sum_{c \mid d} \frac{\mu(c)}{c} s\left(\frac{h c}{(k, c)}, \frac{k}{(k, c)}\right) \\
& =2 \pi \sum_{c \mid d} \mu(c) s(h c, k) / c
\end{aligned}
$$

wherein the last step we used the fact that $s(q h, q k)=s(h, k)$ for any positive integer $q$ (cf. [27], Theorem 1).

To prove Subrahmanyam's identity, we have by (1.2) and Lemma 4.2

$$
\begin{aligned}
\sum_{b(\bmod d)} s(h+b k, d k) & =\frac{1}{2 \pi} \sum_{\substack{r=1 \\
r \neq 0(\bmod d k)}}^{\infty} \frac{1}{r} \sum_{b(\bmod d)} \cot \left(\frac{\pi r h}{d k}+\frac{\pi r b}{d}\right) \\
& =\frac{1}{2 \pi} \sum_{\substack{r=1 \\
r \neq 0(\bmod d k)}}^{\infty} \frac{d}{r} \cot \left(\frac{\pi r h}{k(r, d)}\right) .
\end{aligned}
$$

On writing $(r, d)=s, r=t s, d=u s$ where $(t, u)=1$, this reduces to

$$
\begin{aligned}
\frac{1}{2 \pi} \sum_{\substack{t=1, d=u s \\
t \neq 0(\bmod u k) \\
(t, k)=1}}^{\infty} \frac{u}{t} \cot \left(\frac{\pi t h}{k}\right) & =\frac{1}{2 \pi} \sum_{u s=d} u \sum_{\substack{t=1,(t, u)=1 \\
t \neq 0(\bmod k)}}^{\infty} \cot \left(\frac{\pi t h}{k}\right) \\
& =\sum_{u s=d} u \sum_{m \in=u} \frac{\mu(m) s(h m, k)}{m} \\
& =\sum_{m \mid d} \mu(m) s(h m, k) \sum_{m \mid \frac{d}{m}} n \\
& =\sum_{m \mid d} \mu(m) s(h m, k) \sigma(d / m)
\end{aligned}
$$

wherein the above we used Lemma 4.3. This proves Theorem 4.1 (and as noted earlier Theorem 4.2 also):

5. Hardy sums in terms of Dedekind sums. In this section, we express each one of Hardy sums explicitly in terms of $s(h, k)$. In stating Hardy sums and Hardy's reciprocity theorems we will use the notation of B. C. Berndt and L. A. Goldberg. [12]. If $h$ and $k$ are integers with $k>0$, the Hardy sums are defined by

$$
\begin{aligned}
& S(h, k)=\sum_{j=1}^{k-1}(-1)^{j+1+[h j / k]} \\
& s_{1}(h, k)=\sum_{j=1}^{k}(-1)^{[h j / k]}\left(\left(\frac{j}{k}\right)\right) \\
& s_{2}(h, k)=\sum_{j=1}^{k}(-1)^{j}\left(\left(\frac{h j}{k}\right)\right)\left(\left(\frac{j}{k}\right)\right) \\
& s_{3}(h, k)=\sum_{j=1}^{k}(-1)^{j}\left(\left(\frac{h j}{k}\right)\right) \\
& s_{4}(h, k)=\sum_{j=1}^{k-1}(-1)^{[h j / k]}, \\
& s_{5}(h, k)=\sum_{j=1}^{k}(-1)^{j+[h j / k]}\left(\left(\frac{j}{k}\right)\right)
\end{aligned}
$$

THEOREM 5.1 (Explicit Formulae). Let $(h, k)=1$. Then

and

(5.7) Each one of $S(h, k)\left(h+k\right.$ even), $s_{1}(h, k)$ ( $h$ odd $), s_{2}(h, k)$ ( $k$ odd), $s_{3}(h, k)\left(k\right.$ even), $s_{4}(h, k)\left(h\right.$ even) and $s_{5}(h, k)(h+k$ odd $)$ is zero.

Proof. The proof depends on some simple properties of $((x)), s(h, k)$, some well-known results due to $\mathbf{H}$. Rademacher and A. L. Whiteman [27] (who used only elementary arguments) and the following basic observation

$$
(-1)^{[x]}=2((x))-4\left(\left(\frac{x}{2}\right)\right) \text { if } x \text { is not an integer. }
$$

To prove this, observe that if $x$ is not an integer, then

$$
\begin{aligned}
2((x))-4\left(\left(\frac{x}{2}\right)\right) & =2\left(x-[x]-\frac{1}{2}\right)-4\left(\frac{x}{2}-\left[\frac{x}{2}\right]-\frac{1}{2}\right) \\
& \equiv 1-2[x]+4\left[\frac{x}{2}\right]=1-2[x]+4\left[\frac{[x]}{2}\right]
\end{aligned}
$$




$$
\begin{aligned}
& =\left\{\begin{array}{lll}
1-2[x]+4 \frac{[x]}{2} & \text { if } & {[x] \text { is even }} \\
1-2[x]+4\left(\frac{[x]-1}{2}\right) & \text { if } \quad[x] \text { is odd }
\end{array}\right. \\
& =(-1)^{[x]} .
\end{aligned}
$$

Now to prove (5.1), we have, for $(h, k)=1$ and $h+k$ odd, by (5.8) (5.9)

$$
\begin{aligned}
S(h, k) & =\sum_{j=1}^{k-1}(-1)^{\left[\frac{(n+k) j+k}{k}\right]} \\
& =2 \sum_{j=1}^{k-1}\left(\left(\frac{(h+k) j+k}{k}\right)\right)-4 \sum_{j=1}^{k-1}\left(\left(\frac{(h+k) j+k}{2 k}\right)\right) \\
& =-4 \sum_{j=1}^{k-1}\left(\left(\frac{(h+k) j}{2 k}+\frac{1}{2}\right)\right) .
\end{aligned}
$$

However by H. Rademacher and A. L. Whiteman (cf. [27], eqns. (5.61), (6.1) and (4.31))

$$
\text { (5.10) } \begin{aligned}
-\sum_{j=1}^{k-1}\left(\left(\frac{(h+k) j}{2 k}+\frac{1}{2}\right)\right) & =\sum_{j=1}^{k-1}\left(\left(\frac{(h+k) j}{2 k}\right)\right) \\
& =\sum_{j=1}^{2 k}\left(\left(\frac{j}{2 k}-\frac{1}{2}\right)\right)\left(\left(\frac{(h+k) j}{2 k}\right)\right)-s(h+k, 2 k) \\
& =s(2 h+2 k, 2 k)-s(h+k, 2 k)-s(h+k, 2 k) \\
& =s(h, k)-2 s(h+k, 2 k) .
\end{aligned}
$$

Now it is known due to Dedekind (cf. [27], Theorem 2, $p=2$ ) that

$$
s(h+k, 2 k)=3 s(h, k)-s(2 h, k)-s(h, 2 k)
$$

and thus (5.1) follows from (5.9), (5.10) and (5.11).

To prove (5.2), we have, for $(h, k)=1$ and $h$ even, by (5.8)

$$
\begin{aligned}
s_{1}(h, k) & =\sum_{j=1}^{k-1}(-1)^{[h j / k]}\left(\left(\frac{j}{k}\right)\right) \\
& =2 \sum_{j=1}^{k-1}\left(\left(\frac{h j}{k}\right)\right)\left(\left(\frac{j}{k}\right)\right)-4 \sum_{j=1}^{k-1}\left(\left(\frac{h j}{2 k}\right)\right)\left(\left(\frac{j}{k}\right)\right) \\
& =2 s(h, k)-4 s(h / 2, k)=2 s(h, k)-4 s(h, 2 k) .
\end{aligned}
$$

To prove (5.3), we have, for $k$ even

$$
s_{2}(h, k)=\sum_{\substack{j=1 \\ j=1}}^{k}\left(\left(\frac{h j}{k}\right)\right)\left(\left(\frac{j}{k}\right)\right)-\sum_{\substack{j=1 \\ j \text { odd }}}^{k}\left(\left(\frac{h j}{k}\right)\right)\left(\left(\frac{j}{k}\right)\right)
$$

$$
\begin{aligned}
& =2 \sum_{\substack{j=1 \\
j \text { even }}}^{k}\left(\left(\frac{h j}{k}\right)\right)\left(\left(\frac{j}{k}\right)\right)-\sum_{j=1}^{k}\left(\left(\frac{h j}{k}\right)\right)\left(\left(\frac{j}{k}\right)\right) \\
& =2 \sum_{j=1}^{k / 2}\left(\left(\frac{2 h j}{k}\right)\right)\left(\left(\frac{2 j}{k}\right)\right)-s(h, k) \\
& =2 s(h, k / 2)-s(h, k)=2 s(2 h, k)-s(h, k) .
\end{aligned}
$$

Note that $(h, k)=1$ is not used in the proof of (5.3).

To prove (5.4), we have, for $k$ odd by $\mathrm{H}$. Rademacher and A. L. Whiteman (cf. [27], eqns. (6.1) and (4.4))

$$
\begin{aligned}
s_{3}(h, k) & =\sum_{j=1}^{k-1}(-1)^{j}\left(\left(\frac{h j}{k}\right)\right)=\sum_{j=1}^{k-1}\left(1+(-1)^{j}\right)\left(\left(\frac{h j}{k}\right)\right) \\
& =2 \sum_{j=1}^{(k-1) / 2}\left(\left(\frac{2 h j}{k}\right)\right) \\
& =2 \sum_{j=1}^{k}\left(\left(\frac{j}{k}-\frac{1}{2}\right)\right)\left(\left(\frac{2 h j}{k}\right)\right)-2 s(2 h, k) \\
& =2(s(h, k)-s(2 h, k))-2 s(2 h, k)=2 s(h, k)-4 s(2 h, k) .
\end{aligned}
$$

To prove (5.5), we have, for $h$ odd, by (5.8) and H. Rademacher and A. L. Whiteman (cf. [27], eqn. (6.4))

$$
\begin{aligned}
s_{4}(h, k) & =\sum_{j=1}^{k-1}(-1)^{[h j k j]}=2 \sum_{j=1}^{k-1}\left(\left(\frac{h j}{k}\right)\right)-4 \sum_{j=1}^{k-1}\left(\left(\frac{h j}{2 k}\right)\right) \\
& =-4 \sum_{j=1}^{k-1}\left(\left(\frac{h j}{2 k}\right)\right)=-4(s(2 h, 2 k)-2 s(h, 2 k)) \\
& =-4 s(h, k)+8 s(h, 2 k) .
\end{aligned}
$$

To prove (5.6), we have by (5.8)

$$
\begin{aligned}
s_{5}(h, k) & =\sum_{j=1}^{k-1}(-1)^{[j(h+k) / k]}\left(\left(\frac{j}{k}\right)\right) \\
& =\sum_{j=1}^{k-1}\left(2\left(\left(\frac{j(h+k)}{k}\right)\right)-4\left(\left(\frac{j(h+k)}{2 k}\right)\right)\right)\left(\left(\frac{j}{k}\right)\right) \\
& =2 s(h, k)-4 s\left(\frac{h+k}{2}, k\right)=2 s(h, k)-4 s(h+k, 2 k) .
\end{aligned}
$$

Now (5.11) completes the proof.

To prove (5.7), we have

$$
S(h, k)=\sum_{j=1}^{k-1}(-1)^{j+1+[h j / k]}=\sum_{j=1}^{k-1}(-1)^{k-j+1+[h(k-j) / k]}
$$




$$
=\sum_{j=1}^{k-1}(-1)^{k-j+1+h-[h j / k]-1}=(-1)^{h+k-1} S(h, k) .
$$

Since $h+k$ is even, this proves that $S(h, k)=0$. The proofs of the remaining assertions in (5.7) are similar. This completes the proof of Theorem 5.1.

6. Hardy's reciprocity theorems. As mentioned in the introduction, G. H. Hardy [19] proved some reciprocity theorems in detail and stated, at the end of his paper, 11 more reciprocity theorems with clear indications of proofs. In this section, we give elementary proofs of 5 reciprocity theorems due to Hardy (capsulized into 4), using the notation of Section 5 .

TheOREM 6.1. Let $h$ and $k$ be coprime positive integers. Then

$$
S(h, k)+S(k, h)=1 \text { if } h+k \text { is odd, }
$$

$$
\begin{gathered}
s_{1}(h, k)-2 s_{2}(k, h)=\frac{1}{2}-\frac{1}{2}\left(\frac{1}{h k}+\frac{k}{h}\right) \quad \text { if } \quad h \text { is even, } \\
2 s_{3}(h, k)-s_{4}(k, h)=1-\frac{h}{k} \quad \text { if } \quad k \text { is odd, } \\
s_{5}(h, k)+s_{5}(k, h)=\frac{1}{2}-\frac{1}{2 h k} \quad \text { if } \quad h \text { and } k \text { are odd. }
\end{gathered}
$$

Remark 6.1. The reciprocity theorems (6.1) through (6.4) appear in Hardy's [19] list respectively as equations (viii), (vii), (vi)-(vi') and (ix) on pages $122-123$. B. C. Berndt [8] deduced (6.1), (6.2) and (6.3) and L. A. Goldberg [18] deduced (6.4) from Berndt's transformation formulae [8]. For various proofs of Theorem 6.1, which do not depend on transformation theory, we refer to B. C. Berndt and L. A. Goldberg [12], U. Dieter [16], B. Davis and the author [3], T. M. Apostol and T. H. Vu [1] and B. C. Berndt and U. Dieter [10]. Also, all the reciprocity theorems (6.1)-(6.4) are, in fact, special cases of "three-term relations" that have been discovered by L. A. Goldberg [18]. For elementary proofs of these "three-term relations", we refer to $M$. Pettet and the author [23]. Finally, the other reciprocity theorems of Hardy were deduced by L. A. Goldberg [18] from Berndt's transformation formulae and the reciprocity theorem for generalized Dedekind sums [25].

Proof of Theorem 6.1. Since $s(q h, q k)=s(h, k)$ for any positive integer $q$, we have, by (1.1), for arbitrary positive integers $h$ and $k$

$$
s(h, k)+s(k, h)=-\frac{1}{4}+\frac{1}{12}\left(\frac{h}{k}+\frac{k}{h}+\frac{d^{2}}{h k}\right)
$$

where $d=(h, k)$

Now (6.1) follows from (5.1) and (6.5). The proofs of (6.2) through (6.4) are similar.
7. Series representations for Hardy sums. In this section, we give elementary proofs of infinite and finite series representations for Hardy sums which are originally due to B. C. Berndt and L. A. Goldberg [12].

Theorem 7.1. Let $(h, k)=1$. Then

(7.1) $S(h, k)=\frac{4}{\pi} \sum_{r=1}^{\infty} \frac{1}{2 r-1} \tan \left(\frac{\pi h(2 r-1)}{2 k}\right)$

$$
=\frac{1}{k} \sum_{r=1}^{k-1} \tan \left(\frac{\pi h(2 r-1)}{2 k}\right) \cot \left(\frac{\pi(2 r-1)}{k}\right) \text { if } h+k \text { is odd },
$$

$$
\begin{aligned}
s_{1}(h, k) & =-\frac{2}{\pi} \sum_{\substack{r=1 \\
2 r-1}}^{\infty} \frac{1}{2 r-1} \cot \left(\frac{\pi h(2 r-1)}{2 k}\right) \\
& =-\frac{1}{2 k} \sum_{\substack{r=1 \\
r \neq(k+1) / 2}}^{k-1} \cot \left(\frac{\pi h(2 r-1)}{2 k}\right) \cot \left(\frac{\pi(2 r-1)}{2 k}\right)
\end{aligned}
$$

$$
\begin{aligned}
s_{2}(h, k) & =-\frac{1}{2 \pi} \sum_{\substack{r=1 \\
2 r=0(\bmod k)}}^{\infty} \frac{1}{r} \tan \left(\frac{\pi h r}{k}\right) \\
& =-\frac{1}{4 k} \sum_{r=1}^{k-1} \tan \left(\frac{\pi h r}{k}\right) \cot \left(\frac{\pi r}{k}\right)
\end{aligned}
$$

$$
\begin{aligned}
s_{3}(h, k) & =\frac{1}{\pi} \sum_{r=1}^{\infty} \frac{1}{r} \tan \left(\frac{\pi h r}{k}\right) \\
& =\frac{1}{2 k} \sum_{r=1}^{k-1} \tan \left(\frac{\pi h r}{k}\right) \cot \left(\frac{\pi r}{k}\right) \text { if } k \text { is odd, }
\end{aligned}
$$

if $h$ is odd and $k$ is even,$$
s_{4}(h, k)=\frac{4}{\pi} \sum_{r=1}^{\infty} \frac{1}{2 r-1} \cot \left(\frac{\pi h(2 r-1)}{2 k}\right)
$$$$
=\frac{1}{k} \sum_{r=1}^{k-1} \cot \left(\frac{\pi h(2 r-1)}{2 k}\right) \cot \left(\frac{\pi(2 r-1)}{2 k}\right) \text { if } h \text { is odd, }
$$$$
s_{5}(h, k)=\frac{2}{\pi} \sum_{\substack{r=1 \\ 2 r-1}}^{\infty} \frac{1}{2 r-1} \tan \left(\frac{\pi h(2 r-1)}{2 k}\right)
$$

$$
=\frac{1}{2 k} \sum_{\substack{k=1 \\ r \neq(k+1) / 2}}^{k-1} \tan \left(\frac{\pi h(2 r-1)}{2 k}\right) \cot \left(\frac{\pi(2 r-1)}{2 k}\right)
$$

if $h$ and $k$ are odd. 
Proof. To prove the first part of (7.1), we first note that by (5.11)

$$
s(h, k)=4 s(h, k)-8 s(h+k, 2 k)
$$

if $(h, k)=1$ and $h+k$ is odd. Hence by Dedekind's representation (1.2)

$$
S(h, k)=\frac{4}{2 \pi}\left(\sum_{\substack{r=1 \\ r \neq 0(\bmod k)}}^{\infty} \frac{\cot (\pi h r / k)}{r}-2 \sum_{\substack{r=1 \\ r \neq 0(\bmod 2 k)}}^{\infty} \frac{\cot (\pi(h+k) r / 2 k)}{r}\right) .
$$

Now on splitting the second series on the right into two according as $r$ is even or odd, we obtain the assertion (since we do not have absolute convergence, a limiting argument is necessary). The second part of (7.1) follows from our basic Lemma 2.1. The proofs of (7.2) through (7.6) are similar.

8. Subrahmanyam's and Knopp's results for Hardy sums. Our explicit formulae for Hardy sums combined with Subrahmanyam's identity yield the following

THEOREM 8.1. Let $(h, k)=1$ and $b$ be an odd positive integ Then (8.1) $\quad \sum_{m=0}^{b-1} S(h+2 m k, b k)=\sum_{d \mid b} \mu(d) S(h d, k) \sigma\left(\frac{b}{d}\right) \quad$ if $h+k$ is odd.

Further if $y_{i}=2$ or 1 according as $i=1,4,5$ or 2,3 and $h$ even for $i=1, k$ even for $i=2, k$ odd for $i=3, h$ odd for $k=4$ and both $h$ and $k$ odd for $i=5$, then

$$
\sum_{m=0}^{b-1} s_{i}\left(h+y_{i} m k, b k\right)=\sigma(n) s_{i}(h, k) \quad \text { for } \quad 1 \leqslant i \leqslant 5
$$

The identity (8.1) is due to L. A. Goldberg (cf. [18], Chapter 6).

Theorem 8.1 combined with our inversion formula given in Lemma 4.1 gives the following result due to L. A. Goldberg (cf. [18], Chapter 8).

THEOREM 8.2. Under the notation of Theorem 4.1, we have

$$
\sum_{\substack{a d x=1 \\ d>0}} \sum_{r=0}^{d-1} S(a h+2 r k, d k)=\sigma(n) S(h, k)
$$

and

$$
\sum_{\substack{a d=n \\ d>0}} \sum_{r=0}^{d-1} s_{i}\left(a h+y_{i} r k, d k\right)=\sigma(n) s_{i}(h, k) \quad \text { for } \quad 1 \leqslant i \leqslant 5
$$

9. A remark on Dedekind-Rademacher sums. L. A. Goldberg (cf. [18], Theorem 8.1), while discussing the other reciprocity theorems of Hardy,

considered the Dedekind-Rademacher sum $s(h, k ; x, y)[25]$ defined by

$$
s(h, k ; x, y)=\sum_{\mu(\bmod k)}\left(\left(\frac{h(\mu+y)}{k}+x\right)\right)\left(\left(\frac{\mu+y}{k}\right)\right) .
$$

Using Berndt's transformation formulae, Goldberg expressed $s\left(h, k ; \frac{1}{2}, \frac{1}{2}\right)$, in case $(h, k)=1$, explicitly in terms of Hardy sums.

It may be of interest to note that, by a simple elementary argument, we have, for any two positive integers $h$ and $k$, the following curious result

$$
s\left(h, k ; \frac{1}{2}, \frac{1}{2}\right)=2 s(h, k)-s(h, 2 k)-s(2 h, k) \text {. }
$$

In fact, by the well-known result $\left(\left(x+\frac{1}{2}\right)\right)=((2 x))-((x))$ (cf. [27], eqn. (2.41)), we have

$$
\begin{aligned}
s\left(h, k ; \frac{1}{2}, \frac{1}{2}\right)= & \sum_{\mu(\bmod k)}\left(\left(\frac{h(\mu+1 / 2)}{k}+\frac{1}{2}\right)\right)\left(\left(\frac{\mu+1 / 2}{k}\right)\right) \\
= & \sum_{\mu(\operatorname{mad} k)}\left(\left(\left(\frac{2 h(2 \mu+1)}{2 k}\right)\right)-\left(\left(\frac{h(2 \mu+1)}{2 k}\right)\right)\right)\left(\left(\frac{2 \mu+1}{2 k}\right)\right) \\
= & \sum_{\mu(\bmod 2 k)}\left(\left(\left(\frac{2 h \mu}{2 k}\right)\right)-\left(\left(\frac{h \mu}{2 k}\right)\right)\right)\left(\left(\frac{\mu}{2 k}\right)\right) \\
& \quad-\sum_{\mu(\bmod k)}\left(\left(\left(\frac{2 h 2 \mu}{2 k}\right)\right)-\left(\left(\frac{h 2 \mu}{2 k}\right)\right)\right)\left(\left(\frac{2 \mu}{2 k}\right)\right) \\
& =s(2 h, 2 k)-s(h, 2 k)-(s(2 h, k)-s(h, k))
\end{aligned}
$$

which gives at once $(9.1)$.

\section{References}

[1] T. M. Apostol and T. H. Vu, Elementary proofs of Berndt's reciprocity laws, Pacific Journ. Math. 98 (1982), pp. $17-23$.

[2] - - Identities for sums of Dedekind type, Journ. of Number Theory 14 (1982), pp. 391-396.

[3] B. C. Berndt, $A$ new proof of the reciprocity theorem for Dedekind sums, Elem. Math. 29 (1974), pp. 93-94.

[4] - Problem 73-26, A double summation, SIAM Rev. 16 (1974), pp, 555-557.

[5] - The evaluation of certain classes of nonabsolutely convergent double series, SIAM Journ. Math. Anal. 6 (1975), pp. 966-977.

[6] - Dedekind sums and a paper of G. H. Hardy, Journ. London Math. Soc., (2), 13 (1976), pp. 129-136.

[7] - Reciprocity theorems for Dedekind sums and generalizations, Advances in Math. 23 (1977), pp, 285-316.

[8] - Analytic Eisenstein series, theta-functions, and series relations in the spirit of Ramanujan Journ. Reine Angew. Math. 303/304 (1978), pp. 332-365.

[9] - Solution to problem E2719, Amer. Math. Monthly 86 (1979), p. 787. 
[10] B. C. Berndt and U. Dieter, Sums involving the greatest integer function and RiemannStieljes integration, Journ, Reine Angew. Math. 337 (1982), pp. 208-220.

[11] B. C. Berndt and R. J. Evans. Problem E2758, Amer. Math. Monthly 87 (1980), pp. 404-405.

[12] B. C. Berndt and L. A. Goldberg, Analytic properties of arithmetic sums arising in the theory of the classical theta-functions, SIAM Journ. Math. Anal. 15 (1984), pp. 143-150

[13] T. J. I'. A. Bromwich, An introduction to the theory of infinite series, 2nd edition, Macmillan and Co., London 1926.

[14] B. Davis and R. Sitaramachandrarao, Arithmetical properties of Hardy sums, in preparation.

[15] R. Dedekind, Erlatuterungen zu der Riemannschen Fragmenten iber die Grenzfalle der elliptischen Funktionen, Gesammelte Math. Werke 1, Braunschweig 1930, pp. 159-173.

[16] U. Dieter, Cotangent sums, a further generalization of Dedekind sums, Journ. Number Theory 18 (1984), pp. 289-305.

[17] L. A. Goldberg, An elementary proof of the Peterson-Knopp theorem on Dedekind sums, ibid. 12 (1980), pp. 541-542.

[18] - Transformations of theta-functions and analogues of Dedekind sums, Thesis, University of Illinois, Urbana, 1981

[19] G. H. Hardy, On certain series of discontinuous functions connected with the modular functions, Quart. Journ. Math. 36 (1905), pp. 93-123=Collected papers, Vol. IV, pp. 362 392. Clarendon Press, Oxford 1969.

[20] M. I. Knopp, Hecke operators and an identity for the Dedekind sums, Journ. Number Theory 12 (1980), pp, 2-9.

[21] D. H. Lehmer, Euler constants for arithmetical progressions, Acta Arith. 27 (1975), pp. $125-142$.

[22] L. A. Parson and K. H. Rosen, Hecke operators and Lambert series, Math. Scand. 49 (1981), pp. 5-14.

[23] M. Pettet and R. Sitaramachandrarao, Three-term relations, for Hardy sums, Journ. Number Theory 25 (1987).

[24] H. Rademacher, Egy Reciprocitásképletröl a Modulfüggevenyek Elméletéböl, Mat. Fiz Lapok 40 (1933), pp. 24-34.

[25] - Some remarks on certain generalized Dedekind sums, Acta Arith. 9 (1964), pp. 97-105.

[26] H. Rademacher and E. Grosswald, Dedekind sums. Carus Mathematical Monograph, No. 16, Math. Assoc. of America, Washington, D. C., 1972.

[27] H. Rademacher and A. L. Whiteman, Theorems on Dedekind sums, Amer. Journ. Math. 63 (1941), pp. 377-407,

[28] P. Subrahmanyam, On sums involving the integer part of $x$, Math. Student 45 (1977), pp. 8-12.

[29] E. T. Whittaker and G. N. Watson, A course of Modern Analysis, 4th edition, Cambridge 1962

DEPARTMENT OF MATHEMATICS

THE UNIVERSTTY OF TOLEDO

Toledo, Ohio 43606, U.S.A.

\section{On unit solutions of the equation $x y z=x+y+z$ in the ring of integers of a quadratic field}

by

R. A. Mollin (Calgary), C. Small (Kingston), K. Varadarajan (Calgaty) and P. G. Walsh (Calgary)*

1. Introduction. This work was inspired by a study of the equation $x y z$ $=x+y+z=1$ which is known to have no solutions in the rational number field $Q$ (see [1], [2] and [3]). In [4] this equation is studied over finite fields, and a precise count is given therein of the number of solutions in the finite fields. It is natural to ask the more general question: What are the solutions of $x y z=x+y+z=u$ where $u$ is a unit in the ring of integers of a number field? Equivalently; what are the solutions of $x y z=x+y+z$ where $x, y, z$ are units in the ring of integers of a number field? It is the purpose of this paper to completely solve this problem in the quadratic number field case.

2. Results. In what follows $U_{K}$ denotes the units of the ring of integers of $K=Q(\sqrt{d})$, where $d$ is a square-free rational integer.

THEOREM. There exist solutions to:

$$
u_{1} u_{2} u_{3}=u_{1}+u_{2}+u_{3}
$$

where $u_{i} \in U_{K}$ for $i=1,2,3$ if and only if $d=-1,2$ or 5 .

A complete classification of the solutions for each $d$ is given in Table 4 following the proof of the theorem.

Proof. First we consider the case $d<0$. If $d \neq-1$ or -3 then $U_{K}$ $=\{ \pm 1\}$ and the equation $(*)$ is clearly not solvable. If $d=-3$ then we claim there are no solutions. Let $w$ denote a primitive 6 th root of unity. Then $u_{i}$ $=w^{l_{i}}$ where $0 \leqslant l_{i} \leqslant 5$. If any two of the $l_{i}$ 's are equal, say $l_{1}=l_{2}$ without loss of generality, then $w^{2 l_{1}+l_{3}}=2 w^{l_{1}}+w^{l_{3}}$ implies $w^{l_{1}+l_{3}}=2+w^{l_{3}-l_{1}}$ whence $w^{l_{1}+l_{3}}-w^{l_{3}-l_{1}}=2$, and so $w^{l_{3}}\left(w^{l_{1}}-w^{-l_{1}}\right)=2$. However for $0 \leqslant l_{1} \leqslant 5$ we get $w^{l_{1}}-w^{-l_{1}}=0$ or $\pm \sqrt{-3}$, which yields a contradiction in

* The first three authors' research is supported by. N.S.E.R.C. Canada, and the fourth author was a senior undergraduate mathematics student at The University of Calgary at the time this paper was written. 